\title{
A further case of Parastrongyliasis (=Angiostrongyliasis) from the eye of a patient in Sri Lanka
}

\author{
R. L. Ihalamulla', *S. D. Fernando', K. H. Weerasena', J. H. Cross², A. S. Dissanaike', C. Fonseka ${ }^{4}$ \\ The Ceylon Journal of Medical Science 2007; 50: 15-17
}

\begin{abstract}
Raw snails and slugs are rarely, if ever, consumed in Sri Lanka. If such mollusks are infected with the 3rd stage larvae of the lungworm parasite Parastrongylus (=Angiostrongylus), human infection can occur and a number of such cases have been encountered in this country. We now report what we believe is the 5 th such infection. All these cases have been from the eye, including the anterior chamber, the vitreous and the retina. In the present case the worm was in the vitreous.
\end{abstract}

\section{Introduction}

A natural, lung parasite of rodents, Parastrongylus (=Angiostrongylus) cantonensis is commonly found in South and Southeast Asian countries. The primary cause of eosinophilic meningitis or eosinophilic meningoencephalitis in the Asia-Pacific region is the invasion of the central nervous system by the 3 rd stage larva of this worm (1). The eye is another possible site of infection but ocular involvement is rare and it could cause blindness. P. cantonensis has an indirect life cycle and requires an intermediate host to complete it. Different species of molluscs serve as intermediate hosts. Slugs and snails infected with the larvae of $P$. cantonenis are the source of infection to humans. These molluscs are eaten raw or half cooked in some countries in the Southeast Asian region whereby the larva migrates to meninges, eye etc. In Sri Lanka where such raw food is not taken the possible mode of infection is eating salads of raw leaves contaminated with snails or slugs.

\section{Case report}

In January 2007, a 25 year old female from Kirulapone in the district of Colombo, Sri Lanka, presented at a private hospital in Colombo with a "vision of a large worm revolving in circles" of 2 weeks duration. Prior to this, an ophthalmologist and a physician whom she had consulted had dismissed the symptom and advised psychological counseling. On admission her vision was $6 / 60$. Fundus examination revealed a live worm on the surface of the retina of the right eye with multiple retinal tracks. Emergency transparsplana

1. Department of Parasitology, Faculty of Medicine, University of Colombo, Kynsey Road, Colombo 08, Sri Lanka.

2. The Uniformed Services, University of Health Services, Bethesda, Maryland, USA.

3. 28, Welikadewatte, Nawala Road, Rajagiriya, Sri Lanka.

4. Ward 13, Eye Hospital, Colombo 08, Sri Lanka.

* Author for correspondence. E-mail: deepfern@slt.lk. Department of Parasitology, Faculty of Medicine, Kynsey Road, Colombo 8, Sri Lanka. 
vitrectomy was performed and the worm was recovered. Following surgery the vision came back to normal. The worm was sent to the Department of Parasitology, Faculty of Medicine, Colombo for parasitological diagnosis.

The patient has been in the habit of eating salads made of raw lettuce, "gotukola" (Centella asiatica) and carrots almost daily. Her mother gave more details; she keeps the leaves brought from the market, overnight, half immersed in water in a bucket to prevent them from drying, outside the kitchen. The following morning she finds slugs migrated on to the leaves. She washes the leaves in tap water but only occasionally in salt water. There are bandicoots, rats, snails and slugs in their garden and that of their neighbours'.

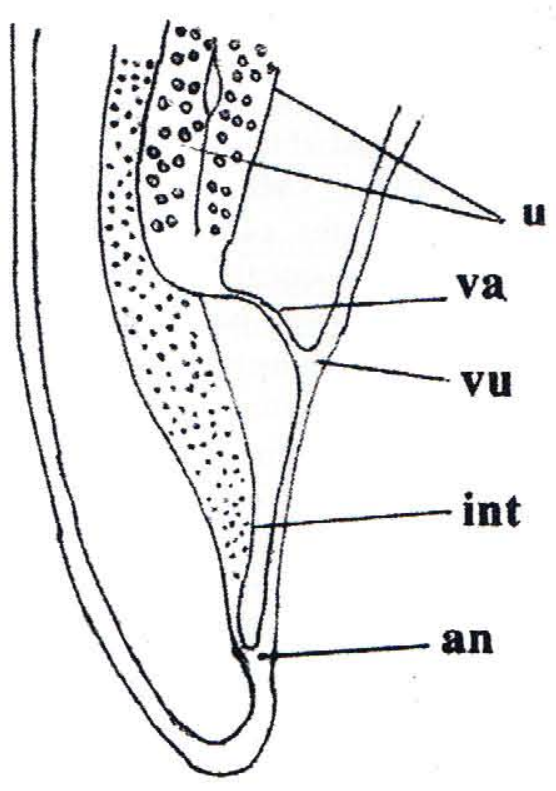

Figure 1. Posterior end of worm - no terminal projection. u: uteri, va: vagina, vu: vulva, int: intestine, an: anus

\section{Description of the worm}

It has fine transverse striations on the cuticle; body length $8.5 \mathrm{~mm}$; width $180 \mu \mathrm{m}$; vulva just anterior to the anus and 109.5 $\mu \mathrm{m}$ from the posterior end of body which shows no terminal projection (Figure 1). Based on morphological features the nematode was identified as an immature female of Parastrongylus sp probably cantonensis which is commonly reported from bandicoots (Bandicota malabarica) in Sri Lanka.

\section{Discussion}

The presence of the natural host the rodent and the intermediate host the mollusc in the house premises of the patient promotes the completion of the life cycle of the Parastrongylus worm thus resulting in a fair population of infected slugs or snails. This increases the chance of human infection. If the leaves are washed in salt water the molluscs will be destroyed. Otherwise when these are cut to prepare salads any infected slugs would be sliced and the larvae will be released into the preparation. Washing leaves in salt water before consuming them raw is a simple and an important preventive measure.

It is important that medical officers and ophthalmologists are made aware of parastrongyliasis - though it is rare considering the serious consequences it could cause. The fact that even an ophthalmologist did misdiagnose the condition in the present case confirms this. Had the infection been ignored, retinal damage would have resulted in serious consequences to the vision of this woman.

The first ocular infection was reported in Sri Lanka in 1925 (2). Subsequently three more cases $(3,4,5)$ have been reported. The present case is the fifth from this country. Cases of ocular infection have also been reported from India(6), Thailand (7) and Vietnam (8). 
All the Parastrongylus (=Angiostrongylus) worms recovered in Sri Lanka were from the eye and not from other parts of the body such as the brain although Alibhoy, et al. (9) reported a case of eosinophilic meningoencephalitis based solely on serological evidence. It is possible that predilection for the eye in cases from Sri Lanka reflects a strain difference.

\section{Acknowledgements}

The authors thank Dr.S.A.S.C. Senanayake of the Department of Parasitology, Faculty of Medicine, Colombo 8, Sri Lanka for helping in the acquisition of information for the report. They also wish to thank Mrs. Himali Gunathilake of the same Department for technical assistance.

\section{References}

1. Cross J.H. Studies on angiostrongyliasis in Eastern Asia and Australia. Taipei: US Naval Medical Research Unit No 2, 1979.

2. Dissanaike A.S., Cross J.H. Ocular parastrongyliasis (=angiostrongyliasis): probable first report of human infection from a patient in Ceylon (Sri Lanka). Parassitologia 2004; 46: 315-316.

3. Durette-Desset M.C., Chabaud A.G., Cassim M.H.S., Ismail M.M. et al. On an infection of a human eye with Parastrongylus (= Angiostrongylus) sp. in Sri Lanka . Journal of Helminthology 1993; 67: 69-72.
4. Wariyapola D., Goonesinghe N., Priyamanne T.H.H., Fonseka C., Ismail M.M. et al. Second case of ocular parastrongyliasis from Sri Lanka. Transactions of the Royal Society of Tropical Medicine and Hygiene 1998; 92: 64-65.

5. Dissanaike A.S., Ihalamulla R.L., Naotunne T. De S., Senarathna T., Withana D.S.R. Third report of ocular parastrongyliasis (angiostrongyliasis) from Sri Lanka. Parassitologia 2001; 43: 95-97.

6. Malhotra S., Mehta D.K., Arora R., Chauhan D. Ray S. et al. Ocular angiostrongyliasis in a child - first case report from India. Journal of Tropical Paediatrics 2006; 52: 223-225.

7. Sawanyawisuth K., Kitthaweesin K., Limpawattana P., Intapan P.M. Tiamkao S. et al. Intraocular angiostrongyliasis: clinical findings, treatments and outcomes. Transactions of the Royal Society of Tropical Medicine and Hygiene 2007; 101: 497-501.

8. Thu T.P., Nguyen N.X., Lan Le T., Kuchle M. Ocular Angiostrongylus cantonensis in a female Vietnamese patient: case report. Klinische Monatsblätter für Augenheilkunde 2002; 219: 892-895.

9. Alibhoy A.T., Senanayaka B., Fernando M.A.H., Amerasekera H.S.U., Wijesekra J.C. A case of eosinophilic meningitis. Ceylon Medical Journal 1999; 44: 173-174. 